\title{
Alignment for Rooted Labeled Caterpillars
}

\author{
Yoshiyuki Ukita \\ Graduate School of Computer Science and Systems Engineering \\ Kyushu Institute of Technology \\ Kawazu 680-4, Iizuka 820-8502, Japan \\ Email: o231014y@mail.kyutech.jp
}

\author{
Takuya Yoshino, Kouichi Hirata* \\ Department of Artificial Intelligence \\ Kyushu Institute of Technology \\ Kawazu 680-4, Iizuka 820-8502, Japan \\ Email: \{yoshino,hirata\}@ai.kyutech.ac.jp
}

\begin{abstract}
A rooted labeled caterpillar (caterpillars, for short) is a rooted labeled tree transformed to a rooted path after removing all the leaves in it. In this paper, we design the algorithm to compute the alignment distance between caterpillars in $O\left(h^{2} \lambda^{3}\right)$ time under the general cost function and in $O\left(h^{2} \lambda\right)$ time under the unit cost function, where $h$ is the maximum height and $\lambda$ is the maximum number of leaves in caterpillars.
\end{abstract}

\section{INTRODUCTION}

$\mathbf{C}$ OMPARING tree-structured data such as HTML and XML data for web mining or RNA and glycan data for bioinformatics is one of the important tasks for data mining. The most famous distance measure [2] between rooted labeled unordered trees (trees, for short) is the edit distance [10]. The edit distance is formulated as the minimum cost of edit operations, consisting of a substitution, a deletion and an insertion, applied to transform a tree to another tree. It is known that the edit distance is always a metric and coincides with the minimum cost of Tai mappings [10].

Unfortunately, the problem of computing the edit distance between trees is MAX SNP-hard [15]. This statement also holds even if trees are binary or the maximum height of trees is at most 3 [1], [4].

A caterpillar (cf. [3]) is a tree transformed to a rooted path after removing all the leaves in it. Whereas the caterpillars are very restricted and simple, there are some cases containing many caterpillars in real dataset, see Table I in Appendix. Recently, Muraka et al. [8] have proposed the algorithm to compute the edit distance between caterpillars in $O\left(h^{2} \lambda^{3}\right)$ time under the general cost function and in $O\left(h^{2} \lambda\right)$ time under the unit cost function, where $h$ is the maximum height and $\lambda$ is the maximum number of leaves in caterpillars ${ }^{1}$. They have also introduced the efficient comparable distances to approximate the edit distance between caterpillars [9].

An alignment distance is an alternative distance measure between trees, introduced by Jiang et al. [5]. The alignment distance between two trees is formulated as the minimum cost of possible alignments (as trees) obtained by first inserting nodes labeled with spaces into two trees so that the resulting trees have the same structure and then overlaying them. In

\footnotetext{
$*^{*}$ The author would like to express thanks for support by Grant-in-Aid for Scientific Research 17H00762, 16H02870 and 16H01743 from the Ministry of Education, Culture, Sports, Science and Technology, Japan.

${ }^{1}$ This time complexity is different from the result in [8], because it contains some errors. See Appendix.
}

operational, the alignment distance is regarded as an edit distance such that every insertion precedes to deletions. Hence, the alignment distance between trees is not always equal to the edit distance and regarded as a variation of the edit distance. Furthermore, Kuboyama [6] has shown that the alignment distance coincides with the minimum cost of less-constrained mappings [7], which is the restriction of the Tai mapping.

As same as the edit distance, the problem of computing the alignment distance between trees is also MAX SNP-hard [5]. On the other hand, it is tractable if the degrees are bounded by some constant [5]. Since a caterpillar is not a bounded-degree tree, it is still open whether or not the problem of computing the alignment distance is tractable,

In this paper, first we point out that there exists a pair of caterpillars whose minimum cost less-constrained mapping is not an isolated-subtree mapping and whose minimum cost Tai mapping is not a less-constrained mapping. Then, we can apply the algorithm of computing neither the isolatedsubtree distance or its variations [12], [13], [14], [16] nor the edit distance [8] to compute the alignment distance between caterpillars.

Next, we design the algorithm to compute the alignment distance between caterpillars in $O\left(h^{2} \lambda^{3}\right)$ time under the general cost function and in $O\left(h^{2} \lambda\right)$ time under the unit cost function. Here, it is necessary to adopt the edit distance for multisets $(c f .,[9])$ to compute the alignment distance between sets of leaves. Furthermore, as same as the edit distance [8], we point out the structural restriction of caterpillars provides the limitation of tractable computing of the alignment distance for unordered trees.

\section{PRELIMINARIES}

In this section, we prepare the notions necessary to discuss the later sections.

A tree $T$ is a connected graph $(V, E)$ without cycles, where $V$ is the set of vertices and $E$ is the set of edges. We denote $V$ and $E$ by $V(T)$ and $E(T)$. The size of $T$ is $|V|$ and denoted by $|T|$. We sometime denote $v \in V(T)$ by $v \in T$. We denote an empty tree $(\emptyset, \emptyset)$ by $\emptyset$. A rooted tree is a tree with one node $r$ chosen as its root. We denote the root of a rooted tree $T$ by $r(T)$.

Let $T$ be a rooted tree such that $r=r(T)$ and $u, v, w \in$ $T$. We denote the unique path from $r$ to $v$, that is, the tree 
$\left(V^{\prime}, E^{\prime}\right)$ such that $V^{\prime}=\left\{v_{1}, \ldots, v_{k}\right\}, v_{1}=r, v_{k}=v$ and $\left(v_{i}, v_{i+1}\right) \in E^{\prime}$ for every $i(1 \leq i \leq k-1)$, by $U P_{r}(v)$.

The parent of $v(\neq r)$, which we denote by $\operatorname{par}(v)$, is its adjacent node on $U P_{r}(v)$ and the ancestors of $v(\neq r)$ are the nodes on $U P_{r}(v)-\{v\}$. We say that $u$ is a child of $v$ if $v$ is the parent of $u$ and $u$ is a descendant of $v$ if $v$ is an ancestor of $u$. We denote the set of children of $v$ by $\operatorname{ch}(v)$. We call a node with no children a leaf and denote the set of all the leaves in $T$ by $l v(T)$.

The degree of $v$, denoted by $d(v)$, is the number of children of $v$, and the degree of $T$, denoted by $d(T)$, is $\max \{d(v)$ $v \in T\}$. The height of $v$, denoted by $h(v)$, is $\max \left\{\left|U P_{v}(w)\right| \mid\right.$ $w \in l v(T[v])\}$, and the height of $T$, denoted by $h(T)$, is $\max \{h(v) \mid v \in T\}$.

We use the ancestor orders $<$ and $\leq$, that is, $u<v$ if $v$ is an ancestor of $u$ and $u \leq v$ if $u<v$ or $u=v$. We say that $w$ is the least common ancestor of $u$ and $v$, denoted by $u \sqcup v$, if $u \leq w, v \leq w$ and there exists no node $w^{\prime} \in T$ such that $w^{\prime} \leq w, u \leq w^{\prime}$ and $v \leq w^{\prime}$. Let $T$ be a rooted tree $(V, E)$ and $v$ a node in $T$. A complete subtree of $T$ at $v$, denoted by $T[v]$, is a rooted tree $T^{\prime}=\left(V^{\prime}, E^{\prime}\right)$ such that $r\left(T^{\prime}\right)=v$, $V^{\prime}=\{u \in V \mid u \leq v\}$ and $E^{\prime}=\left\{(u, w) \in E \mid u, w \in V^{\prime}\right\}$

We say that $u$ is to the left of $v$ in $T$ if $\operatorname{pre}(u) \leq \operatorname{pre}(v)$ for the preorder number pre in $T$ and $\operatorname{post}(u) \leq \operatorname{post}(v)$ for the postorder number post in $T$. We say that a rooted tree is ordered if a left-to-right order among siblings is given; unordered otherwise. We say that a rooted tree is labeled if each node is assigned a symbol from a fixed finite alphabet $\Sigma$. For a node $v$, we denote the label of $v$ by $l(v)$, and sometimes identify $v$ with $l(v)$. In this paper, we call a rooted labeled unordered tree a tree simply. Furthermore, we call a set of trees a forest.

As the restricted form of trees, we introduce a rooted labeled caterpillar (caterpillar, for short) as follows, which this paper mainly deals with.

Definition 1 (Caterpillar (cf., [3])): We say that a tree is a caterpillar if it is transformed to a rooted path after removing all the leaves in it. For a caterpillar $C$, we call the remained rooted path a backbone of $C$ and denote it by $b b(C)$.

It is obvious that $r(C)=r(b b(C))$ and $V(C)=b b(C) \cup$ $l v(C)$ for a caterpillar $C$, that is, every node in a caterpillar is either a leaf or an element of the backbone.

Next, we introduce an edit distance and a Tai mapping between trees.

Definition 2 (Edit operations for trees [10]): The edit operations of a tree $T$ are defined as follows, see Figure 1.

1) Substitution: Change the label of the node $v$ in $T$.

2) Deletion: Delete a node $v$ in $T$ with parent $v^{\prime}$, making the children of $v$ become the children of $v^{\prime}$. The children are inserted in the place of $v$ as a subset of the children of $v^{\prime}$. In particular, if $v$ is the root in $T$, then the result applying the deletion is a forest consisting of the children of the root.

3) Insertion: The complement of deletion. Insert a node $v$ as a child of $v^{\prime}$ in $T$ making $v$ the parent of a subset of the children of $v^{\prime}$.

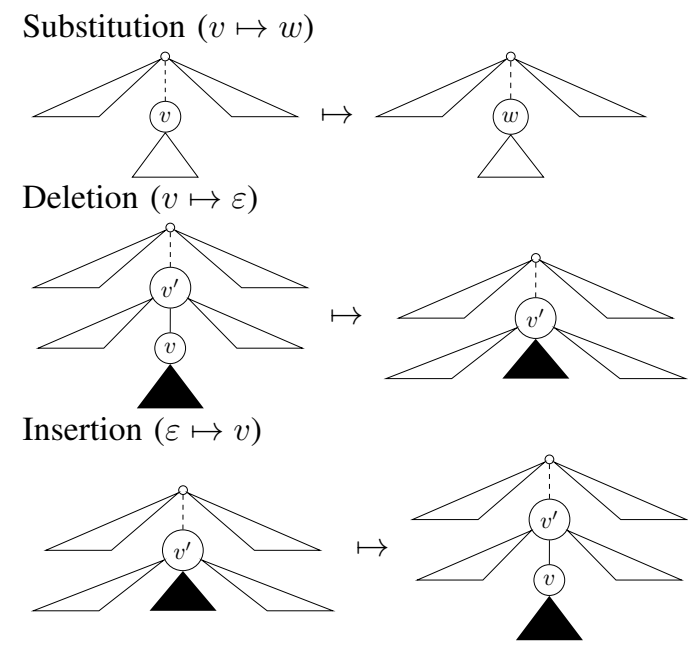

Fig. 1. Edit operations for trees.

Let $\varepsilon \notin \Sigma$ denote a special blank symbol and define $\Sigma_{\varepsilon}=$ $\Sigma \cup\{\varepsilon\}$. Then, we represent each edit operation by $\left(l_{1} \mapsto l_{2}\right)$, where $\left(l_{1}, l_{2}\right) \in\left(\Sigma_{\varepsilon} \times \Sigma_{\varepsilon}-\{(\varepsilon, \varepsilon)\}\right)$. The operation is a substitution if $l_{1} \neq \varepsilon$ and $l_{2} \neq \varepsilon$, a deletion if $l_{2}=\varepsilon$, and an insertion if $l_{1}=\varepsilon$. For nodes $v$ and $w$, we also denote $(l(v) \mapsto l(w))$ by $(v \mapsto w)$.

We define a cost function $\gamma:\left(\Sigma_{\varepsilon} \times \Sigma_{\varepsilon} \backslash\{(\varepsilon, \varepsilon)\}\right) \mapsto \mathbf{R}^{+}$on pairs of labels. For $(v, w) \in V\left(T_{1}\right) \times V\left(T_{2}\right)$, we also denote $\gamma(l(v), l(w))$ by $\gamma(v, w)$ simply.

We often constrain a cost function $\gamma$ to be a metric, that is, $\gamma\left(l_{1}, l_{2}\right) \geq 0, \gamma\left(l_{1}, l_{2}\right)=0$ iff $l_{1}=l_{2}, \gamma\left(l_{1}, l_{2}\right)=\gamma\left(l_{2}, l_{1}\right)$ and $\gamma\left(l_{1}, l_{3}\right) \leq \gamma\left(l_{1}, l_{2}\right)+\gamma\left(l_{2}, l_{3}\right)$. In particular, we call the cost function that $\gamma\left(l_{1}, l_{2}\right)=1$ if $l_{1} \neq l_{2}$ a unit cost function.

Definition 3 (Edit distance for trees [10]): For a cost function $\gamma$, the cost of an edit operation $e=l_{1} \mapsto l_{2}$ is given by $\gamma(e)=\gamma\left(l_{1}, l_{2}\right)$. The cost of a sequence $E=e_{1}, \ldots, e_{k}$ of edit operations is given by $\gamma(E)=\sum_{i=1}^{k} \gamma\left(e_{i}\right)$. Then, an edit distance $\tau_{\mathrm{TAI}}\left(T_{1}, T_{2}\right)$ between trees $T_{1}$ and $T_{2}$ is defined as follows:

$$
\tau_{\text {TAI }}\left(T_{1}, T_{2}\right)=\min \left\{\begin{array}{l|l}
\gamma(E) & \begin{array}{l}
E \text { is a sequence } \\
\text { of edit operations } \\
\text { transforming } T_{1} \text { to } T_{2}
\end{array}
\end{array}\right\} .
$$

Definition 4 (Tai mapping [10]): Let $T_{1}$ and $T_{2}$ be trees. We say that a triple $\left(M, T_{1}, T_{2}\right)$ is a Tai mapping (a mapping, for short) from $T_{1}$ to $T_{2}$ if $M \subseteq V\left(T_{1}\right) \times V\left(T_{2}\right)$ and every pair $\left(v_{1}, w_{1}\right)$ and $\left(v_{2}, w_{2}\right)$ in $M$ satisfies the following conditions.

1) $v_{1}=v_{2}$ iff $w_{1}=w_{2}$ (one-to-one condition).

2) $v_{1} \leq v_{2}$ iff $w_{1} \leq w_{2}$ (ancestor condition).

We will use $M$ instead of $\left(M, T_{1}, T_{2}\right)$ when there is no confusion denote it by $M \in \mathcal{M}_{\mathrm{TAI}}\left(T_{1}, T_{2}\right)$.

Let $M$ be a mapping from $T_{1}$ to $T_{2}$. Let $I_{M}$ and $J_{M}$ be the sets of nodes in $T_{1}$ and $T_{2}$ but not in $M$, that is, $I_{M}=$ $\left\{v \in T_{1} \mid(v, w) \notin M\right\}$ and $J_{M}=\left\{w \in T_{2} \mid(v, w) \notin M\right\}$. Then, the cost $\gamma(M)$ of $M$ is given as follows.

$$
\gamma(M)=\sum_{(v, w) \in M} \gamma(v, w)+\sum_{v \in I_{M}} \gamma(v, \varepsilon)+\sum_{w \in J_{M}} \gamma(\varepsilon, w) .
$$


Trees $T_{1}$ and $T_{2}$ are isomorphic, denoted by $T_{1} \equiv T_{2}$, if there exists a mapping $M \in \mathcal{M}_{\mathrm{TAI}}\left(T_{1}, T_{2}\right)$ such that $I_{M}=$ $J_{M}=\emptyset$ and $\gamma(M)=0$.

Theorem 1 (Tai [10]): $\tau_{\mathrm{TAI}}\left(T_{1}, T_{2}\right)=\min \{\gamma(M) \mid M \in$ $\left.\mathcal{M}_{\mathrm{TAI}}\left(T_{1}, T_{2}\right)\right\}$.

\section{Alignment Distance}

In this section, we introduce the alignment distance and characterize it by using the variation of Tai mappings.

Definition 5 (Alignment [5]): Let $T_{1}$ and $T_{2}$ be trees. Then, an alignment between $T_{1}$ and $T_{2}$ is a tree $\mathcal{T}$ obtained by the following steps.

1) Insert new nodes labeled by $\varepsilon$ into $T_{1}$ and $T_{2}$ so that the resulting trees $T_{1}^{\prime}$ and $T_{2}^{\prime}$ are isomorphic with ignoring labels and $l(\phi(v)) \neq \varepsilon$ whenever $l(v)=\varepsilon$ for an isomorphism $\phi$ from $T_{1}^{\prime}$ to $T_{2}^{\prime}$ and every node $v \in T_{1}^{\prime}$.

2) Set $\mathcal{T}$ to a tree $T_{1}^{\prime}$ obtained by relabeling a label $l(v)$ for every node $v \in T_{1}^{\prime}$ with $(l(v), l(\phi(v)))$. (Note that $(\varepsilon, \varepsilon) \notin \mathcal{T}$.)

Let $\mathcal{A}\left(T_{1}, T_{2}\right)$ denote the set of all possible alignments between trees $T_{1}$ and $T_{2}$.

For a cost function $\gamma$, the cost of an alignment $\mathcal{T}$, denoted by $\gamma(\mathcal{T})$, is the sum of the costs of all labels in $\mathcal{T}$.

Definition 6 (Alignment distance [5]): Let $T_{1}$ and $T_{2}$ be trees and $\gamma$ a cost function. Then, an alignment distance $\tau_{\text {ALN }}\left(T_{1}, T_{2}\right)$ between $T_{1}$ and $T_{2}$ is defined as follows.

$$
\tau_{\text {ALN }}\left(T_{1}, T_{2}\right)=\min \left\{\gamma(\mathcal{T}) \mid \mathcal{T} \in \mathcal{A}\left(T_{1}, T_{2}\right)\right\}
$$

Also we call an alignment between $T_{1}$ and $T_{2}$ with the minimum cost an optimal alignment and denote it by $\mathcal{A}^{*}\left(T_{1}, T_{2}\right)$.

The notion of the alignment can be easily extended to forests. The only change is that it is now possible to insert a node (as the root) of trees in the forest. We denote the set of all possible alignments between forests $F_{1}$ and $F_{2}$ by $\mathcal{A}\left(F_{1}, F_{2}\right)$ and an optimal alignment by $\mathcal{A}^{*}\left(F_{1}, F_{2}\right)$.

Example 1: For two caterpillars $C_{1}$ and $C_{2}$ illustrated in Figure $2, \mathcal{A}^{*}\left(C_{1}, C_{2}\right)$ is the optimal alignment between $C_{1}$ and $C_{2}$. Also, for two caterpillars $C_{3}$ and $C_{4}$ illustrated in Figure $2, \mathcal{A}^{*}\left(C_{3}, C_{4}\right)$ is the optimal alignment between $C_{3}$ and $C_{4}$. Under the unit cost function, it holds that $\tau_{\mathrm{ALN}}\left(C_{1}, C_{2}\right)=$ 3 and $\tau_{\mathrm{ALN}}\left(C_{3}, C_{4}\right)=3$.
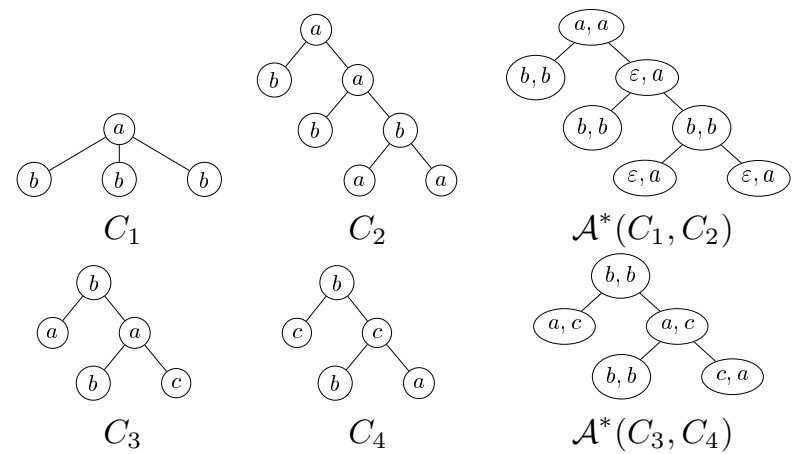

Fig. 2. Two caterpillars $C_{1}, C_{2}, C_{3}$ and $C_{4}$ and the optimal alignments $\mathcal{A}^{*}\left(C_{1}, C_{2}\right)$ and $\mathcal{A}^{*}\left(C_{1}, C_{2}\right)$. in Example 1 .
Next, we introduce the variations of Tai mappings, including the mapping characterizing the alignment distance.

Definition 7 (Variations of Tai mapping): Let $T_{1}$ and $T_{2}$ be trees and $M \in \mathcal{M}_{\mathrm{TAI}}\left(T_{1}, T_{2}\right)$.

1) We say that $M$ is a less-constrained mapping [7], denoted by $M \in \mathcal{M}_{\text {Less }}\left(T_{1}, T_{2}\right)$, if $M$ satisfies the following condition for every $\left(v_{1}, w_{1}\right),\left(v_{2}, w_{2}\right),\left(v_{3}, w_{3}\right) \in M$ :

$$
\left(v_{1} \sqcup v_{2}<v_{1} \sqcup v_{3}\right) \Longrightarrow\left(w_{2} \sqcup w_{3}=w_{1} \sqcup w_{3}\right) \text {. }
$$

Also we define a less-constrained distance $\tau_{\text {LESS }}\left(T_{1}, T_{2}\right)$ as the minimum cost of all the less-constrained mappings, that is:

$$
\tau_{\text {Less }}\left(T_{1}, T_{2}\right)=\min \left\{\gamma(M) \mid M \in \mathcal{M}_{\text {Less }}\left(T_{1}, T_{2}\right)\right\} .
$$

2) We say that $M$ is an isolated-subtree mapping [11] (or a constrained mapping [14]), denoted by $M \in$ $\mathcal{M}_{\text {ILST }}\left(T_{1}, T_{2}\right)$, if $M$ satisfies the following condition for every $\left(v_{1}, w_{1}\right),\left(v_{2}, w_{2}\right),\left(v_{3}, w_{3}\right) \in M$ :

$$
\left(v_{3}<v_{1} \sqcup v_{2}\right) \Longleftrightarrow\left(w_{3}<w_{1} \sqcup w_{2}\right) .
$$

Also we define an isolated-subtree distance $\tau_{\text {ILST }}\left(T_{1}, T_{2}\right)$ as the minimum cost of all the isolated-subtree mappings, that is:

$$
\tau_{\text {ILST }}\left(T_{1}, T_{2}\right)=\min \left\{\gamma(M) \mid M \in \mathcal{M}_{\text {ILST }}\left(T_{1}, T_{2}\right)\right\} .
$$

Theorem 2: Let $T_{1}$ and $T_{2}$ be trees, where $n=$ $\max \left\{\left|T_{1}\right|,\left|T_{2}\right|\right\}$ and $d=\min \left\{d\left(T_{1}\right), d\left(T_{2}\right)\right\}$.

1) It holds that $\tau_{\text {ALN }}\left(T_{1}, T_{2}\right)=\tau_{\text {Less }}\left(T_{1}, T_{2}\right)$ [6]. Also it holds that $\tau_{\mathrm{TAI}}\left(T_{1}, T_{2}\right) \leq \tau_{\mathrm{ALN}}\left(T_{1}, T_{2}\right) \leq \tau_{\mathrm{ILST}}\left(T_{1}, T_{2}\right)$ but the equations always do not hold (cf., [5], [6], [14]).

2) The problem of computing $\tau_{\mathrm{TAI}}\left(T_{1}, T_{2}\right)$ is MAX SNPhard [15]. This statement holds even if both $T_{1}$ and $T_{2}$ are binary, the maximum height of $T_{1}$ and $T_{2}$ is at most 3 or the cost function is the unit cost function [1], [4].

3) The problem of computing $\tau_{\mathrm{ALN}}\left(T_{1}, T_{2}\right)$ is MAX SNPhard. On the other hand, if the degrees of $T_{1}$ and $T_{2}$ are bounded by some constants, then we can compute $\tau_{\text {ALN }}\left(T_{1}, T_{2}\right)$ in polynomial time with respect to $n$ [5].

4) We can compute $\tau_{\text {ILST }}\left(T_{1}, T_{2}\right)$ in $O\left(n^{2} d\right)$ time (cf., [12]).

Example 2: Consider two caterpillars $C_{1}$ and $C_{2}$ in Figure 2 in Example 1 and assume the unit cost function. Then, $M_{1}$ and $M_{2}$ illustrated in Figure 3 are the minimum cost mappings in $\mathcal{M}_{\text {Less }}\left(C_{1}, C_{1}\right)\left(=\mathcal{M}_{\mathrm{TAI}}\left(C_{1}, C_{2}\right)\right)$ and $\mathcal{M}_{\text {ILST }}\left(C_{1}, C_{2}\right)$. Here, it holds that $M_{1} \notin \mathcal{M}_{\text {ILST }}\left(C_{1}, C_{2}\right)$. Then, it holds that $\tau_{\mathrm{TAI}}\left(C_{1}, C_{2}\right)=\tau_{\mathrm{ALN}}\left(C_{1}, C_{2}\right)=3<5=$ $\tau_{\text {ILST }}\left(C_{1}, C_{2}\right)$.

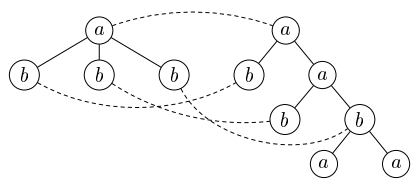

$M_{1}$

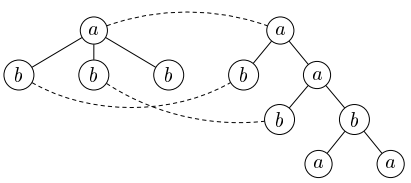

$M_{2}$
Fig. 3. The minimum cost mappings $M_{1} \in \mathcal{M}_{\text {LESS }}\left(C_{1}, C_{2}\right)$ and $M_{2} \in$ $\mathcal{M}_{\text {ILST }}\left(C_{1}, C_{2}\right)$ in Example 2 .

Example 3: Consider two caterpillars $C_{3}$ and $C_{4}$ in Figure 2 in Example 1 and assume the unit cost function. 
Then, $M_{3}$ and $M_{4}$ illustrated in Figure 4 are the minimum cost mappings in $\mathcal{M}_{\mathrm{TAI}}\left(C_{3}, C_{4}\right)$ and $\mathcal{M}_{\mathrm{LESS}}\left(C_{3}, C_{4}\right)$. Here, it holds that $M_{3} \notin \mathcal{M}_{\text {Less }}\left(C_{3}, C_{4}\right)$. Hence, it holds that $\tau_{\mathrm{TAI}}\left(C_{3}, C_{4}\right)=2<3=\tau_{\mathrm{ALN}}\left(C_{3}, C_{4}\right)$.

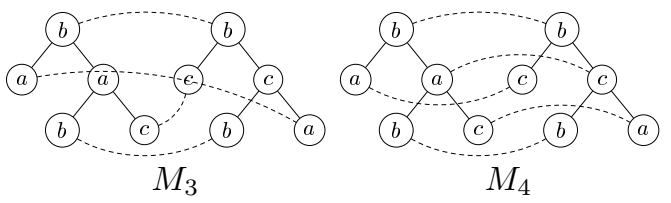

Fig. 4. The minimum cost mappings $M_{3} \in \mathcal{M}_{\mathrm{TAI}}\left(C_{3}, C_{4}\right)$ and $M_{4} \in$ $\mathcal{M}_{\text {Less }}\left(C_{3}, C_{4}\right)$ in Example 3.

Example 2 shows that there exists a pair of caterpillars whose minimum cost less-constrained mapping is not an isolated-subtree mapping. Then, we cannot use the algorithm to compute the isolated-subtree distance between caterpillars [12], [13], [14], [16] to compute their alignment distance. Also Example 3 shows that there exists a pair of caterpillars whose minimum cost Tai mapping is not a less-constrained mapping. Then, we cannot use the algorithm to compute the edit distance between caterpillars [8] to compute their alignment distance. Furthermore, it still remains open whether or not Theorem 2.3 holds for caterpillars.

Hence, in the next section, we discuss the problem of computing the alignment distance between caterpillars.

\section{The Algorithm of Computing Alignment DISTANCE BETWEEN CATERPILLARS}

In this section, we design the algorithm to compute the alignment distance $\tau_{\text {ALN }}$ between caterpillars.

\section{A. Edit distance for multisets}

In order to compute the edit distance between the sets of leaves, it is necessary to introduce an edit distance for multisets on labels occurring in the set of leaves. Then, we prepare the notions of the edit distance for multisets according to [9].

A multiset on an alphabet $\Sigma$ is a mapping $S: \Sigma \rightarrow \mathbf{N}$. For a multiset $S$ on $\Sigma$, we say that $a \in \Sigma$ is an element of $S$ if $S(a)>0$ and denote it by $a \in S$ (like as a standard set). The cardinality of $S$, denoted by $|S|$, is defined as $\sum_{a \in \Sigma} S(a)$.

Definition 8 (Edit operations for multisets): Let $a, b \in \Sigma$ such that $S(a)>0$ and $a \neq b$. Then, a substitution $(a \mapsto b)$ operates $S(a)$ to $S(a)-1$ and $S(b)$ to $S(b)+1$, a deletion $(a \mapsto \varepsilon)$ operates $S(a)$ to $S(a)-1$ and an insertion $(\varepsilon \mapsto b)$ operates $S(b)$ to $S(b)+1$.

Also we assume a cost function $\gamma$ as in Section II.

Definition 9 (Edit distance for multisets): Let $S_{1}$ and $S_{2}$ be multisets on $\Sigma$ and $\gamma$ a cost function. Then, an edit distance $\mu\left(S_{1}, S_{2}\right)$ between $S_{1}$ and $S_{2}$ is defined as follows.

$$
\mu\left(S_{1}, S_{2}\right)=\min \left\{\begin{array}{l|l}
\gamma(E) & \begin{array}{l}
E \text { is a sequence } \\
\text { of edit operations } \\
\text { transforming } S_{1} \text { to } S_{2}
\end{array}
\end{array}\right\} .
$$

For multisets $S_{1}$ and $S_{2}$ on $\Sigma$, we define the difference $S_{1} \backslash S_{2}$ between $S_{1}$ and $S_{2}$ as a multiset satisfying that $\left(S_{1} \backslash\right.$ $\left.S_{2}\right)(a)=\max \left\{S_{1}(a)-S_{2}(a), 0\right\}$ for every $a \in \Sigma$.
Lemma 1 ([9]): Let $\Pi_{1}$ be the set of all the injections from $S_{1}$ to $S_{2}$ when $\left|S_{1}\right| \leq\left|S_{2}\right|$ and $\Pi_{2}$ the set of all the injections from $S_{2}$ to $S_{1}$ when $\left|S_{1}\right|>\left|S_{2}\right|$. Then, we can compute $\mu\left(S_{1}, S_{2}\right)$ as follows:

$$
\mu\left(S_{1}, S_{2}\right)=\left\{\begin{array}{l}
\min _{\pi \in \Pi_{1}}\left\{\sum_{a \in S_{1}} \gamma(a, \pi(a))+\sum_{b \in S_{2} \backslash \pi\left(S_{1}\right)} \gamma(\varepsilon, b)\right\}, \\
\quad \text { if }\left|S_{1}\right| \leq\left|S_{2}\right|, \\
\min _{\pi \in \Pi_{2}}\left\{\sum_{b \in S_{2}} \gamma(\pi(b), b)+\sum_{a \in S_{1} \backslash \pi\left(S_{2}\right)} \gamma(a, \varepsilon)\right\}, \\
\text { otherwise. }
\end{array}\right.
$$

Furthermore, if we adopt the unit cost function, then we can compute $\mu\left(S_{1}, S_{2}\right)$ as follows:

$$
\mu\left(S_{1}, S_{2}\right)=\max \left\{\left|S_{1} \backslash S_{2}\right|,\left|S_{2} \backslash S_{1}\right|\right\}
$$

In this case, $\mu\left(S_{1}, S_{2}\right)$ coincides with a famous bag distance ( $c f$., [2]) between multisets $S_{1}$ and $S_{2}$.

Lemma 2 ([9]): Let $m=\max \left\{\left|S_{1}\right|,\left|S_{2}\right|\right\}$. Then, we can compute $\mu\left(S_{1}, S_{2}\right)$ in $O\left(\mathrm{~m}^{3}\right)$ time under the general cost function. If we adopt the unit cost function, then we can compute $\mu\left(S_{1}, S_{2}\right)$ in $O(m)$ time.

\section{B. Recurrences}

Let $L$ be the set of leaves and $C$ a non-leaf caterpillar. Then, every forest obtained by deleting the root from a caterpillar is one of the forms of $\{C\}, L$ or $L \cup\{C\}$. As same as [8], we denote these forests by $\langle\emptyset \mid C\rangle,\langle L \mid \emptyset\rangle$ and $\langle L \mid C\rangle$, respectively. In particular, we denote an empty forest $\langle\emptyset \mid \emptyset\rangle$ by $\Phi$ simply.

Let $C[v]$ be a caterpillar with the root $v$, where $L(v)$ denotes a (possibly empty) set of leaves as the children of $v$ and $B(v)$ denotes at most one caterpillar of the child $v$. Then, $C[v]$ is one of the forms in Figure 5. Furthermore, by deleting $v$ from $C[v]$, we obtain one of the forests of $\langle\emptyset \mid B(v)\rangle,\langle L(v) \mid \emptyset\rangle$ and $\langle L(v) \mid B(v)\rangle$, respectively.

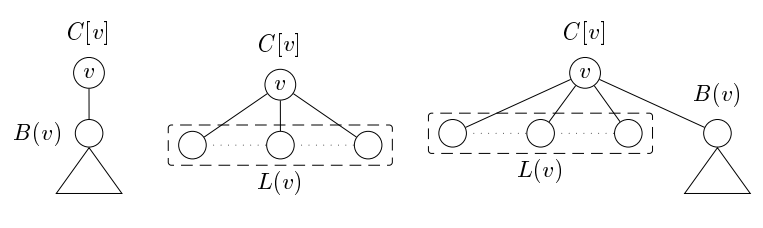

Fig. 5. The representation of a caterpillar $C[v]$.

Figure 6 illustrates the recurrences of computing the alignment distance $\tau_{\mathrm{ALN}}\left(C_{1}[v], C_{2}[w]\right)$ between two caterpillars $C_{1}[v]$ and $C_{2}[w]$. Here, we regard a set $L$ of leaves as a multiset of labels on $\Sigma$ occurring in $L$, which we denote by $\widetilde{L}$. Also $\delta_{\text {ALN }}\left(\left\langle L_{1} \mid B_{1}\right\rangle,\left\langle L_{2} \mid B_{2}\right\rangle\right)$ describes the alignment distance between forests $\left\langle L_{1} \mid B_{1}\right\rangle$ and $\left\langle L_{2} \mid B_{2}\right\rangle$. Furthermore, assume that the forest obtained by deleting $v$ (resp., $w$ ) from $C_{1}[v]$ (resp., $C_{2}[w]$ ) is $\left\langle L_{1}(v) \mid B_{1}(v)\right\rangle$ (resp., $\left\langle L_{2}(w) \mid B_{2}(w)\right\rangle$ ).

Theorem 3: The recurrences in Figure 6 are correct to compute the alignment distance $\tau_{\mathrm{ALN}}\left(C_{1}[v], C_{2}[w]\right)$ between $C_{1}[v]$ and $C_{2}[w]$. 
$\tau_{\mathrm{ALN}}(\emptyset, \emptyset)=0$

$\tau_{\mathrm{ALN}}\left(C_{1}[v], \emptyset\right)=\gamma(v, \varepsilon)+\delta_{\mathrm{ALN}}\left(\left\langle L_{1}(v) \mid B_{1}(v)\right\rangle, \Phi\right)$.

$\tau_{\mathrm{ALN}}\left(\emptyset, C_{2}[w]\right)=\gamma(\varepsilon, w)+\delta_{\mathrm{ALN}}\left(\Phi,\left\langle L_{2}(w) \mid B_{2}(w)\right\rangle\right)$.

$\delta_{\mathrm{ALN}}\left(\left\langle L_{1} \mid C_{1}\right\rangle, \Phi\right)=\sum_{v \in L_{1}} \gamma(v, \varepsilon)+\sum_{v \in C_{1}} \gamma(v, \varepsilon)$.

$\delta_{\mathrm{ALN}}\left(\Phi,\left\langle L_{2} \mid C_{2}\right\rangle\right)=\sum_{w \in L_{2}} \gamma(\varepsilon, w)+\sum_{w \in C_{2}} \gamma(\varepsilon, w)$.

$\delta_{\mathrm{ALN}}\left(\left\langle L_{1} \mid \emptyset\right\rangle,\left\langle L_{2} \mid \emptyset\right\rangle\right)=\mu\left(\widetilde{L_{1}}, \widetilde{L_{2}}\right)$.

(A) $\tau_{\mathrm{ALN}}\left(C_{1}[v], C_{2}[w]\right)$

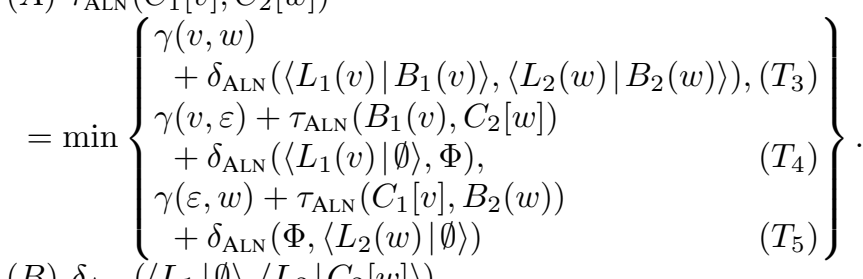

(B) $\delta_{\mathrm{ALN}}\left(\left\langle L_{1} \mid \emptyset\right\rangle,\left\langle L_{2} \mid C_{2}[w]\right\rangle\right)$

$=\min \left\{\begin{array}{c}\gamma(\varepsilon, w) \\ \quad+\delta_{\mathrm{ALN}}\left(\left\langle L_{1} \mid \emptyset\right\rangle,\left\langle L_{2} \cup L_{2}(w) \mid B_{2}(w)\right\rangle\right),\left(F_{4}\right) \\ \min _{v \in L_{1}}\left\{\gamma(v, w)+\delta_{\mathrm{ALN}}\left(\left\langle L_{1} \backslash\{v\} \mid \emptyset\right\rangle,\left\langle L_{2} \mid \emptyset\right\rangle\right)\right\} \\ +\delta_{\mathrm{ALN}}\left(\Phi,\left\langle L_{2}(w) \mid B_{2}(w)\right\rangle\right)\end{array}\right\}$.

$(C) \delta_{\mathrm{ALN}}\left(\left\langle L_{1} \mid C_{1}[v]\right\rangle,\left\langle L_{2} \mid \emptyset\right\rangle\right)$

$=\min \left\{\begin{array}{lr}\gamma(v, \varepsilon) & \\ \quad+\delta_{\mathrm{ALN}}\left(\left\langle L_{1} \cup L_{1}(v) \mid B_{1}(v)\right\rangle,\left\langle L_{2} \mid \emptyset\right\rangle\right), & \left(F_{6}\right) \\ \min _{w \in L_{2}}\left\{\gamma(v, w)+\delta_{\mathrm{ALN}}\left(\left\langle L_{1} \mid \emptyset\right\rangle,\left\langle L_{2} \backslash\{w\} \mid \emptyset\right\rangle\right)\right\} \\ +\delta_{\mathrm{ALN}}\left(\left\langle L_{1}(v) \mid B_{1}(v)\right\rangle, \Phi\right) & \left(F_{7}\right)\end{array}\right\}$

(D) $\delta_{\mathrm{ALN}}\left(\left\langle L_{1} \mid C_{1}[v]\right\rangle,\left\langle L_{2} \mid C_{2}[w]\right\rangle\right)$

$=\delta_{\mathrm{ALN}}\left(\left\langle L_{1} \mid \emptyset\right\rangle,\left\langle L_{2} \mid \emptyset\right\rangle\right)+\tau_{\mathrm{ALN}}\left(C_{1}[v], C_{2}[w]\right)$.

Fig. 6. The recurrences of computing the alignment distance $\tau_{\mathrm{ALN}}\left(C_{1}[v], C_{2}[w]\right)$ between $C_{1}[v]$ and $C_{2}[w]$.

Proof: The recurrences of $\left(T_{0}\right),\left(T_{1}\right),\left(T_{2}\right),\left(F_{1}\right),\left(F_{2}\right)$ and $\left(F_{3}\right)$ are obvious.

First, consider the recurrences for $\tau_{\mathrm{ALN}}$. Let $\mathcal{T}$ be the optimal alignment (tree) $\mathcal{A}^{*}\left(C_{1}[v], C_{2}[w]\right)$. Then, for the label in $\mathcal{T}$, one of the following four cases holds.

1) $(v, w)$ is a label in $\mathcal{T}$.

2) $(v, \varepsilon)$ and $\left(v^{\prime}, w\right)$ are labels in $\mathcal{T}$.

3) $(\varepsilon, w)$ and $\left(v, w^{\prime}\right)$ are labels in $\mathcal{T}$.

4) $(v, \varepsilon)$ and $(\varepsilon, w)$ are labels in $\mathcal{T}$.

It is not necessary to consider the case 4) because the resulting alignment to delete the two nodes and then add $(v, w)$ as the new root, which is the case 1), has a smaller cost.

For the case 1), the root of $\mathcal{T}$ is $(v, w)$. By the forms of $C_{1}[v]$ and $C_{2}[w]$, it holds that $\tau_{\mathrm{ALN}}\left(C_{1}[v], C_{2}[w]\right)=$ $\gamma(v, w)+\delta_{\mathrm{ALN}}\left(\left\langle L_{1}(v) \mid B_{1}(v)\right\rangle,\left\langle L_{2}(w) \mid B_{2}(w)\right\rangle\right)$, which is the recurrence $\left(T_{3}\right)$.

For the case 2), the root of $\mathcal{T}$ is $(v, \varepsilon)$. By the form of $C_{1}[v]$, since $\left|B_{1}(v)\right| \geq 2, B_{1}(v)$, not $L_{1}(v)$, contains the node $v^{\prime}$ corresponding to $w$ in $C_{2}[w]$. Then, $\mathcal{T}$ contains a label $\left(v^{\prime \prime}, \varepsilon\right)$ for every $v^{\prime \prime} \in L_{1}(v)$. Hence, it holds that $\tau_{\mathrm{ALN}}\left(C_{1}[v], C_{2}[w]\right)=$ $\gamma(v, \varepsilon)+\tau_{\mathrm{ALN}}\left(B_{1}(v), C_{2}[w]\right)+\delta_{\mathrm{ALN}}\left(\left\langle L_{1}(v) \mid \emptyset\right\rangle, \Phi\right)$, which is the recurrence $\left(T_{4}\right)$. The case 3$)$ is similar to the case 2$)$, which is the recurrence $\left(T_{5}\right)$.
Next, consider the recurrences for $\delta_{\mathrm{ALN}}$.

Let $\mathcal{F}$ be the optimal alignment (forest) $\mathcal{A}^{*}\left(\left\langle L_{1} \mid \emptyset\right\rangle,\left\langle L_{2}\right|\right.$ $\left.\left.C_{2}[w]\right\rangle\right)$. Then, for the label in $\mathcal{F}$, one of the following two cases holds.

1) $(\varepsilon, w)$ is a label in $\mathcal{F}$.

2) $(v, w)$ for some $v \in L_{1}$ is a label in $\mathcal{F}$.

For the case 1), by deleting $w$ from $C_{2}[w],\left\langle L_{2} \mid C_{2}[w]\right\rangle$ is transformed to $\left\langle L_{2} \cup L_{2}(w) \mid B_{2}(w)\right\rangle$. Hence, it holds that $\delta_{\mathrm{ALN}}\left(\left\langle L_{1} \mid \emptyset\right\rangle,\left\langle L_{2} \mid C_{2}[w]\right\rangle\right)=\gamma(\varepsilon, w)+\delta_{\mathrm{ALN}}\left(\left\langle L_{1} \mid \emptyset\right\rangle,\left\langle L_{2} \cup\right.\right.$ $\left.L_{2}(w)\left|B_{2}(w)\right\rangle\right)$, which is the recurrence $\left(F_{4}\right)$.

For the case 2), once $(v, w)$ for some $v \in L_{1}$ becomes a label in $\mathcal{F}$, every label in $\mathcal{F}$ for every $w^{\prime} \in\left\langle L_{2}(w) \mid B_{2}(w)\right\rangle$ is always of the form $\left(\varepsilon, w^{\prime}\right)$. Also the labels concerned with leaves except $v \in L_{1}$ in $\mathcal{F}$ can be computed as $\delta_{\mathrm{ALN}}\left(\left\langle L_{1} \backslash\{v\}\right|\right.$ $\left.\emptyset\rangle,\left\langle L_{2} \mid \emptyset\right\rangle\right)$. Hence, by selecting $v \in L_{1}$ with the minimum cost, we obtain the recurrence $\left(F_{5}\right)$.

By using the same discussion, for the case that $\mathcal{F}$ is the optimal alignment (forest) $\mathcal{A}^{*}\left(\left\langle L_{1} \mid C_{1}[v]\right\rangle,\left\langle L_{2} \mid \emptyset\right\rangle\right)$, we obtain the recurrences $\left(F_{6}\right)$ and $\left(F_{7}\right)$.

Let $\mathcal{F}$ be the optimal alignment (forest) $\mathcal{A}^{*}\left(\left\langle L_{1}\right|\right.$ $\left.\left.C_{1}[v]\right\rangle,\left\langle L_{2} \mid C_{2}[w]\right\rangle\right)$. Since $\left|C_{1}[v]\right| \geq 2$ and $\left|C_{2}[w]\right| \geq 2$, $\mathcal{F}$ contains labels for the alignment of $C_{1}[v]$ and $C_{2}[w]$ and that of $L_{1}$ and $L_{2}$. Hence, it holds that $\delta_{\mathrm{ALN}}\left(\left\langle L_{1} \mid C_{1}[v]\right\rangle,\left\langle L_{2}\right|\right.$ $\left.\left.C_{2}[w]\right\rangle\right)=\delta_{\mathrm{ALN}}\left(\left\langle L_{1} \mid \emptyset\right\rangle,\left\langle L_{2} \mid \emptyset\right\rangle+\tau_{\mathrm{ALN}}\left(C_{1}[v], C_{2}[w]\right)\right.$, which is the recurrence $\left(F_{8}\right)$.

Example 4: Consider two caterpillars $C_{1}$ and $C_{2}$ in Figure 2 in Example 1 and assume the unit cost function. By applying the recurrences in Figure 6, we obtain that the alignment distance $\tau_{\mathrm{ALN}}\left(C_{1}, C_{2}\right)$ between $C_{1}$ and $C_{2}$ is 3 illustrated in Figure 7. Here, we represent a multiset as a sequence enclosed by "[" and "]" and a caterpillar as a termlike representation with "[" and "]", that is, $C_{1}=a[b, b, b]$ and $C_{2}=a[b, a[b, b[a, a]]]$.

$$
\begin{aligned}
& \tau_{\mathrm{ALN}}\left(C_{1}, C_{2}\right) \\
= & \underbrace{\gamma(a, a)}_{=0}+\delta_{\mathrm{ALN}}(\langle[b, b, b] \mid \emptyset\rangle,\langle[b] \mid a[b, b[a, a]]\rangle) \\
= & \underbrace{\gamma(\varepsilon, a)}_{=1}+\delta_{\mathrm{ALN}}(\langle[b, b, b] \mid \emptyset\rangle,\langle[b, b] \mid b[a, a]\rangle) \\
= & 1+\underbrace{\gamma(b, b)}_{=0}+\delta_{\mathrm{ALN}}(\langle[b, b] \mid \emptyset\rangle,\langle[b, b] \mid \emptyset\rangle) \\
& +\underbrace{\left.\left.\delta_{\mathrm{ALN}}(\Phi,\langle[a, a]| \emptyset\}\right\rangle\right)}_{=0} \\
= & +\underbrace{\mu([b, b],[b, b])}_{=1}+\underbrace{\gamma(\varepsilon, a)}_{=1}+\underbrace{\gamma(\varepsilon, a)}_{=1}
\end{aligned}
$$

$=3$.

Fig. 7. The result of computing $\tau_{\mathrm{ALN}}\left(C_{1}, C_{2}\right)$ in Example 4 .

Example 5: Consider two caterpillars $C_{3}=a[a, d[b, c]]$ and $C_{4}=a[c, e[b, a]]$ in Figure 2 in Example 1 and assume the unit cost function. By applying the recurrences in Figure 6, we obtain that the alignment distance $\tau_{\mathrm{ALN}}\left(C_{3}, C_{4}\right)$ between $C_{3}$ and $C_{4}$ is 3 illustrated in Figure 8. 


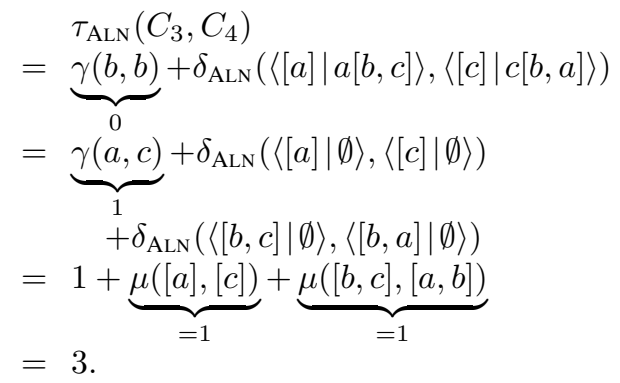

Fig. 8. The result of computing $\tau_{\mathrm{ALN}}\left(C_{3}, C_{4}\right)$ in Example 5 .

\section{Algorithm and time complexity}

Let $C_{1}[v]$ and $C_{2}[w]$ be caterpillars. Then, we denote $b b\left(C_{1}[v]\right)$ by a sequence $v_{1}, \ldots, v_{n}$ such that $v_{n}=v$ and $\operatorname{par}\left(v_{i}\right)=v_{i+1}(1 \leq i \leq n-1)$ and $b b\left(C_{2}[w]\right)$ by a sequence $w_{1}, \ldots, w_{m}$ such that $w_{m}=w$ and $\operatorname{par}\left(w_{j}\right)=w_{j+1}(1 \leq j \leq$ $m-1)$. In this case, we denote by $b b\left(C_{1}[v]\right)=\left[v_{1}, \ldots, v_{n}\right]$ and $b b\left(C_{2}[w]\right)=\left[w_{1}, \ldots, w_{m}\right]$. Also we use the same notations of $L_{1}\left(v_{i}\right)$ and $B_{1}\left(v_{i}\right)$ for $1 \leq i \leq n$ and $L_{2}\left(w_{j}\right)$ and $B_{2}\left(w_{j}\right)$ for $1 \leq j \leq m$.

Based on the recurrences in Figure 6, Algorithm 1 illustrates the algorithm to compute the alignment distance $\tau_{\text {ALN }}\left(C_{1}, C_{2}\right)$ between caterpillars $C_{1}$ and $C_{2}$. Here, the statement " $v \leftarrow$ (A)" means to substitute the value of computing the right side of the recurrence (A) to $v$, for example.

Theorem 4: Let $C_{1}$ and $C_{2}$ be caterpillars, where $h=$ $\max \left\{h\left(C_{1}\right), h\left(C_{2}\right)\right\}$ and $\lambda=\max \left\{\left|l v\left(C_{1}\right)\right|,\left|l v\left(C_{2}\right)\right|\right\}$. Then, we can compute the alignment distance $\tau_{\text {ALN }}\left(C_{1}, C_{2}\right)$ between $C_{1}$ and $C_{2}$ in $O\left(h^{2} \lambda^{3}\right)$ time. Furthermore, if we adopt the unit cost function, then we can compute it in $O\left(h^{2} \lambda\right)$ time.

Proof: Let $b b\left(C_{1}\right)=\left[v_{1}, \ldots, v_{n}\right]$ and $b b\left(C_{2}\right)=$ $\left[w_{1}, \ldots, w_{m}\right]$. Then, it is obvious that $h\left(C_{1}\right)=n+1$ and $h\left(C_{2}\right)=m+1$, so it holds that $m \leq h-1$ and $n \leq h-1$.

The algorithm of computing $\tau_{\mathrm{ALN}}\left(C_{1}, C_{2}\right)$ calls every pair $\left(v_{i}, w_{j}\right) \in b b\left(C_{1}\right) \times b b\left(C_{2}\right)$ just once. When computing $\delta_{\mathrm{ALN}}\left(\left\langle L_{1}\left(v_{i-1}\right) \mid C_{1}\left[v_{i}\right]\right\rangle,\left\langle L_{2}\left(w_{j-1}\right) \mid C_{2}\left[w_{j}\right]\right\rangle\right)$ for $2 \leq i \leq n$ and $2 \leq j \leq m$, it is possible to construct multisets $S_{1}=$ $\widetilde{L_{1}\left(v_{1}\right)} \sqcup \cdots \sqcup L_{1} \widetilde{\left(v_{i-1}\right)}$ and $S_{2}=\widetilde{L_{2}\left(w_{1}\right)} \sqcup \cdots \sqcup L_{2} \widetilde{\left(w_{j-1}\right)}$ and compute the edit distance $\mu\left(S_{1}, S_{2}\right)$ between multisets in the worst case. By Lemma 2, we can compute it in $O\left(\lambda^{3}\right)$ time under the general cost function and in $O(\lambda)$ time under the unit cost function.

Hence, the total running time of computing $\tau_{\mathrm{ALN}}\left(C_{1}, C_{2}\right)$ under the general cost function is described as follows:

$$
\sum_{i=1}^{n} \sum_{j=1}^{m} O\left(\lambda^{3}\right)=O\left(\lambda^{3}\right) m n \leq O\left(\lambda^{3}\right)(h-1)^{2}=O\left(h^{2} \lambda^{3}\right) .
$$

By replacing $O\left(\lambda^{3}\right)$ with $O(\lambda)$, this time complexity is reduced to $O\left(h^{2} \lambda\right)$ time under the unit cost function.

Theorem 4 also claims that the structural restriction of caterpillars provides the limitation of tractable computing the alignment distance for unordered trees as follows. We say that a tree is a generalized caterpillar if it is transformed

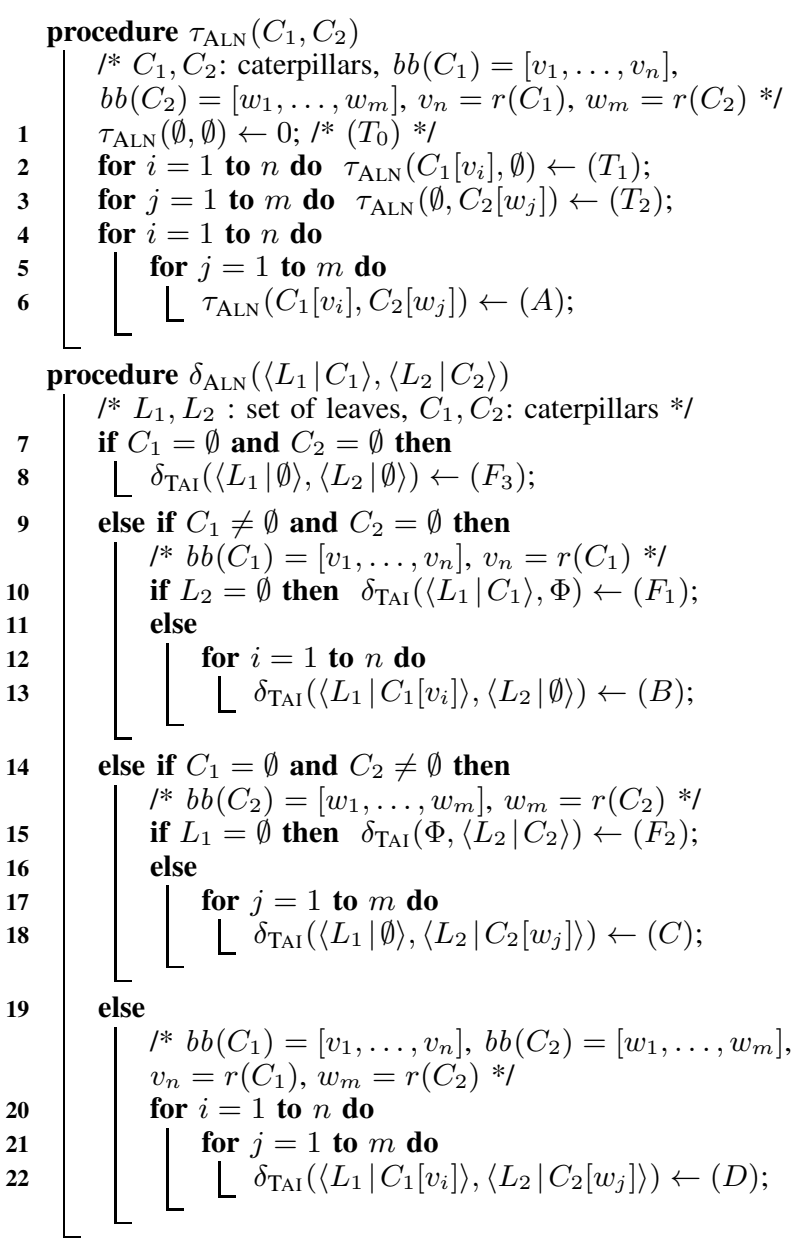

Algorithm 1: $\tau_{\mathrm{ALN}}\left(C_{1}, C_{2}\right)$

to a caterpillar after removing all the leaves in it. Then, the following theorem also holds as a corollary of [8].

Theorem 5 ( $c f .,[1],[4])$ : The problems of computing the alignment distance $\tau_{\text {ALN }}$ between generalized caterpillars are MAX SNP-hard, even if the maximum height is at most 3 and the cost function is the unit cost function.

Proof: It is straightforward from the proof of Corollary 4.3 in [1] or Theorem 1 in [4] and because the Tai mapping constructed in their proof is a less-constrained mapping.

\section{CONCLUSION AND Future WORKS}

In this paper, we have designed the algorithm to compute the alignment distance $\tau_{\mathrm{ALN}}$ between caterpillars in $O\left(h^{2} \lambda^{3}\right)$ time under the general cost function and in $O\left(h^{2} \lambda\right)$ time under the unit cost function.

It is an important future work to implement the algorithms and then give experimental results to compute $\tau_{\mathrm{ALN}}$, with comparing the results of $\tau_{\mathrm{TAI}}$ in [8] with those of $\tau_{\mathrm{ALN}}$. Since the proof in Theorem 4 is rough, it is possible to improve the time complexity, together with that of computing the edit distance between multisets under the general cost function (Lemma 2), which is also a future work. 


\section{REFERENCES}

[1] T. Akutsu, D. Fukagawa, M. M. Halldórsson, A. Takasu, K. Tanaka: Approximation and parameterized algorithms for common subtrees and edit distance between unordered trees, Theoret. Comput. Sci. 470, 10-22 (2013). https://doi.org/10.1016/j.tcs.2012.11.017.

[2] M. M. Deza, E. Deza: Encyclopedia of distances (4th ed.) Springer (2016). https://doi.org/10.1007/978-3-662-52844-0.

[3] J. A. Gallian: A dynamic survey of graph labeling, Electorn. J. Combin., DS6 (2018).

[4] K. Hirata, Y. Yamamoto, T. Kuboyama: Improved MAX SNP-hard results for finding an edit distance between unordered trees, Proc. CPM'11, LNCS 6661, 402-415 (2011). https://doi.org/10.1007/978-3-642-214585 .34.

[5] T. Jiang, L. Wang, K. Zhang: Alignment of trees - an alternative to tree edit, Theoret. Comput. Sci. 143, 137-148 (1995). https://doi.org/10.1016/0304-3975(95)80029-9.

[6] T. Kuboyama: Matching and learning in trees, Ph.D thesis, University of Tokyo (2007)

[7] C. L. Lu, Z.-Y. Su, C. Y. Yang: A new measure of edit distance between labeled trees, Proc. COCOON'01, LNCS 2108, 338-348 (2001). https://doi.org/10.1007/3-540-44679-6_37.

[8] K. Muraka, T. Yoshino, K. Hirata: Computing edit distance between rooted labeled caterpillars, Proc. FedCSIS'18, 245-252 (2018). http://dx.doi.org/10.15439/2018F179.

[9] K. Muraka, T. Yoshino, K. Hirata: Vertical and horizontal distance to approximate edit distance for rooted labeled caterpillars, $\quad$ Proc. ICPRAM'19, 590-597 (2019). https://dx.doi.org/10.5220/0007387205900597.

[10] K.-C. Tai: The tree-to-tree correction problem, J. ACM 26, 422-433 (1979). https://doi.org/10.1145/322139.322143.

[11] J. T. L. Wang, K. Zhang: Finding similar consensus between trees: An algorithm and a distance hierarchy, Pattern Recog. 34, 127-137 (2001). https://doi.org/10.1016/50031-3203(99)00199-5.

[12] Y. Yamamoto, K. Hirata, T. Kuboyama: Tractable and intractable variations of unordered tree edit distance, Internat. J. Found. Comput. Sci. 25, 307-330 (2014). https://doi.org/10.1142/50129054114500154.

[13] T. Yoshino, K. Hirata: Tai mapping hierarchy for rooted labeled trees through common subforest, Theory Comput. Sys. 60, 759-783 (2017). https://doi.org/10.1007/s00224-016-9705-1.

[14] K. Zhang: A constrained edit distance between unordered labeled trees, Algorithmica 15, 205-222 (1996). https://doi.org/10.1007/BF01975866.

[15] K. Zhang, T. Jiang: Some MAX SNP-hard results concerning unordered labeled trees, Inform. Process. Lett. 49, 249-254 (1994). https://doi.org/10.1016/0020-0190(94)90062-0.

[16] K. Zhang, J. Wang, D. Shasha: On the editing distance between undirected acyclic graphs, Internat. J. Found. Comput. Sci. 7, 45-58 (1996). https://doi.org/10.1142/S0129054196000051

\section{APPENDIX}

In this appendix, we point out the number of caterpillars in real data and revise the result for the edit distance between caterpillars in [8].

\section{A. Caterpillars in real data}

Table I, which is represented in [8], illustrates the number of caterpillars in $\mathrm{N}$-glycans and all glycans from $\mathrm{KEGG}^{2}$, CSLOGS $^{3}$, dblp ${ }^{4}$, and SwissProt, TPC-H, Auction, Nasa, Protein and University from UW XML Repository ${ }^{5}$. Here, \#cat is the number of caterpillars and \#data is the total number of data. For $D \in\{$ Auction, Nasa, Protein, University $\}, D^{-}$ denotes the trees obtained by deleting the root for every tree in $D$. Since one tree in $D$ produces some trees in $D^{-}$, the total

\footnotetext{
${ }^{2}$ Kyoto Encyclopedia of Genes and Genomes, http://www.kegg.jp/

${ }^{3}$ http://www.cs.rpi.edu/ zaki/www-new/pmwiki.php/Software/Software

${ }^{4}$ http://dblp.uni-trier.de/

${ }^{5} \mathrm{http} / / /$ aiweb.cs.washington.edu/research/projects/xmltk/xmldata /www/repository.html
}

number of trees in $D^{-}$is greater than that of $D$. Hence, there are some cases containing many caterpillars in real dataset.

TABLE I

THE NUMBER OF CATERPILLARS IN N-GLYCANS AND ALL GLYCANS FROM KEGG, CSLOGS, DBLP, SWISSPROT, TPC-H, AUCTION, UNIVERSITY, PROTEIN AND NASA.

\begin{tabular}{lrrr}
\hline dataset & \#cat & \#data & $\%$ \\
\hline N-glycans & 514 & 2,142 & 23.996 \\
all glycans & 8,005 & 10,704 & 74.785 \\
CSLOGS & 41,592 & 59,691 & 69.679 \\
dblp & $5,154,295$ & $5,154,530$ & 99.995 \\
SwissProt & 6,804 & 50,000 & 13.608 \\
TPC-H & 86,805 & 86,805 & 100.000 \\
\hline Auction & 0 & 37 & 0 \\
Nasa & 0 & 2,430 & 0 \\
Protein & 0 & 262,625 & 0 \\
University $^{\text {Anion }}$ & 0 & 6,738 & 0 \\
\hline Auction $^{-}$ & 259 & 259 & 100.000 \\
Nasa $^{-}$ & 21,245 & 27,921 & 76.089 \\
Protein $^{-}$ & $1,874,703$ & $2,204,068$ & 85.057 \\
University $^{-}$ & 74,638 & 79,213 & 94.224 \\
\hline
\end{tabular}

\section{$B$. The revision of the edit distance for caterpillars}

Muraka et al. [8] have designed the algorithm to compute the edit distance $\tau_{\mathrm{TAI}}\left(C_{1}, C_{2}\right)$. Then, they have pointed out that its time complexity is $O\left(h^{2} \lambda^{2}\right)$ time, where $h=$ $\max \left\{h\left(C_{1}\right), h\left(C_{2}\right)\right\}$ and $\lambda=\max \left\{\left|l v\left(C_{1}\right)\right|,\left|l v\left(C_{2}\right)\right|\right\}$.

Note that their recurrence between the set of leaves is based on the string edit distance. However, as similar as the alignment distance in this paper, in order to compute the edit distance between the set of leaves, it is necessary to adopt the edit distance for multisets.

Let $s(L)$ be the string representation of the set $L$ of leaves and $\sigma$ the string edit distance. Then, Muraka et al. [8] have introduced the following recurrence to compute $\tau_{\mathrm{TAI}}\left(C_{1}, C_{2}\right)$ :

$$
\delta_{\mathrm{TAI}}\left(\left\langle L_{1} \mid \emptyset\right\rangle,\left\langle L_{2} \mid \emptyset\right\rangle\right)=\sigma\left(s\left(L_{1}\right), s\left(L_{2}\right)\right) .
$$

On the other hand, as stated above, it is necessary to replace this recurrence with the following recurrence to compute $\tau_{\text {TAI }}\left(C_{1}, C_{2}\right)$ as same as Figure 6:

$$
\delta_{\mathrm{TAI}}\left(\left\langle L_{1} \mid \emptyset\right\rangle,\left\langle L_{2} \mid \emptyset\right\rangle\right)=\mu\left(\widetilde{L_{1}}, \widetilde{L_{2}}\right) .
$$

Consider the time complexity of $O\left(h^{2} \lambda^{2}\right)$ presented in [8]. The part $O\left(\lambda^{2}\right)$ follows from the time complexity of computing the string edit distance between the set of (all the) leaves in two caterpillars. On the other hand, by replacing the recurrence as above, it is necessary to revise this part based on the time complexity of computing the multiset edit distance, that is, revise to $O\left(\lambda^{3}\right)$ under the general cost function and $O(\lambda)$ under the unit cost function by Lemma 2. Furthermore, we can improve the proof of [8] to the similar proof of Theorem 4.

Hence, we can compute $\tau_{\mathrm{TAI}}\left(C_{1}, C_{2}\right)$ in $O\left(h^{2} \lambda^{3}\right)$ time under the general cost function and $O\left(h^{2} \lambda\right)$ time under the unit cost function, which is the same result of $\tau_{\mathrm{ALN}}\left(C_{1}, C_{2}\right)$. 